\title{
Pathological Sub Types and Diagnostic Protocols of Stroke among Adult Patients Admitted to Jimma University Medical Center, South West Ethiopia
}

Ginenus Fekadu ${ }^{1 *}$, Legesse Chelkeba ${ }^{2}$, Tsegaye Melaku² and Elsah Tegene ${ }^{3}$

${ }^{1}$ Department of Pharmacy, College of Health Sciences, Wollega University, Ethiopia

${ }^{2}$ Department of Pharmacy, Institute of health, Jimma University, Ethiopia

${ }^{3}$ Department of Internal Medicine, Institute of Medical Science, Jimma University, Ethiopia

\begin{abstract}
Background: In the modern approach in treating patients with strokes requires early Computed Tomography (CT) scan as it is imperative to diagnose whether the stroke is ischemic or hemorrhagic. Clinical diagnosis of stroke does not always correlate with imaging; this is even worse when narrowed to clinical diagnosis of specific stroke subtype, creating a high potential for patient morbidity and mortality.
\end{abstract}

Patients and methods: Prospective observational study was carried at stroke unit of Jimma University Medical Center from March 10-July 10, 2017

Result: Of the total 116 patients, 61 patients $(52.6 \%)$ had CT scan of the brain performed, whereas the remaining $(47.4 \%)$ of patients were evaluated clinically alone to have stroke. Using World health organization (WHO) criteria, $51.7 \%$ patients had ischemic stroke while $48.3 \%$ had hemorrhagic, with Intracerebral Hemorrhage (ICH) and Subarachnoid Hemorrhage (SAH) accounting for $44.0 \%$ and $4.3 \%$, respectively. The median time to perform CT scan after hospital arrival was 4 days. The most common site of lesion of brain of hemorrhage stroke patients was basal ganglia $(48.4 \%)$ followed by parietal lobe $(29.0 \%)$ and ventricular $(29.0 \%)$. The most common site of brain infarct was parietal lobe (33.3\%) and cortical $(33.3 \%)$, followed by basal ganglia (23.3\%) and caudate nucleus $(20.0 \%)$. On CT scan majority of hemorrhagic and ischemic stroke patients had Intraparenchymal Bleed (IPB) only (61.3\%) and right hemisphere involvement $(26.7 \%)$ respectively. The predominant stroke etiology as Trial of Org 10172 in Acute Stroke Treatment (TOAST) criteria was undetermined etiology, 26 (43.3\%), mainly because of incomplete evaluation.

Conclusion: Etiologic investigation for stroke was infrequently performed by the lack of systematic cardiological examinations and brain imaging, most of the time for financial reasons and unavailability of the instruments. It is therefore, pertinent that CT scan/ MRI should be incorporated in stroke diagnosis and management where possible.

Keywords: Stroke Diagnosis; CT scan; JUMC; Ethiopia

\section{Introduction}

The data from the Global Burden of Diseases (GBD) showed that the leading CVD cause of death and disability in 2010 in sub-Saharan Africa and other Low and Middle Income Countries (LMICs) was stroke [1-3]. Stroke in young adults has a special significance in developing countries, as it affects the most economically productive group of the society [4] and even it is associated with higher mortality in this age group [5-7]. The proportion of Hemorrhagic Stroke (HS) is higher and greater case fatality in African and other LMICs than in high income countries $[1,8,9]$. Although this disparity is often described to racial or genetic factors, it may actually be due to differences in risk factor burden and associations across these population [8].

Accurate stroke diagnosis and subtype confirmation plays a big role in patient management and prognosis [10]. Although clinical features cannot reliably distinguish Ischemic Stroke (IS) from Intracerebral Hemorrhage (ICH), several clinical signs increase the likelihood of ICH rather than IS: such as coma, neck stiffness, seizures at onset of neurologic deficit, diastolic blood pressure greater than $110 \mathrm{mmHg}$ vomiting and headache are among the few [11].

In the modern approach in treating patients with strokes requires early Computed Tomography (CT) scan as it is imperative to diagnose whether the stroke is ischemic or hemorrhagic [2]. (CT) has enhanced stroke management by confirming the diagnosis, readily differentiating stroke subtypes and excluding stroke mimics like tumors, extra-axial lesions, infections and other stroke-like conditions. In addition to CT scan, Magnetic Resonance Imaging (MRI) remain the standard technique for determining topographic distribution and delineating damaged brain regions [12]. Wrong clinical diagnosis has a high significance for patient outcome [10].

The increasing burden of this chronic disease in Ethiopia poses a challenge to the health care system [13]. Ethiopian patients suffer stroke at a relatively young age, and the proportion of hemorrhagic stroke is higher than in western countries [14]. The burden of ischemic and hemorrhagic stroke varies between regions and over time in Ethiopia as well [15]. Under-diagnosing of hypertension and other risk factors and delayed presentation at the hospital are the major challenges to address [13].

Although the burden of stroke in sub-Saharan Africa, including Ethiopia is increasing, there are few local hospital based studies on stroke in Ethiopia $[14,16,17]$. Like other developing countries resources for stroke care and rehabilitation are deficient in Ethiopia [2]. In addition, significant delays during the pre-hospital or in-hospital phases of care augmented by insufficient management of cases in the country is also making prognosis of stoke patients very poor [2]. The paucity of

*Corresponding author: Ginenus Fekadu, Department of Pharmacy, College of Health Sciences, Wollega University, Ethiopia, Tel: +251917733383, E-mail: take828pharm@gmail.com

Received July 04, 2018; Accepted July 30, 2018; Published July 31, 2018

Citation: Fekadu G, Chelkeba L, Melaku T, Tegene E (2018) Pathological Sub Types and Diagnostic Protocols of Stroke among Adult Patients Admitted to Jimma University Medical Center, South West Ethiopia. J Neurol Neurophysiol 9: 466 doi:10.4172/2155-9562.1000466

Copyright: $\odot 2018$ Fekadu G, et al. This is an open-access article distributed under the terms of the Creative Commons Attribution License, which permits unrestricted use, distribution, and reproduction in any medium, provided the original author and source are credited. 
data specific to the Ethiopian setting is limiting the formulation of an appropriate response [13].

Given the high burden of Cerebrovascular Accident (CVA) and associated inadequately equipped health settings, identifying and mitigating the risk of can be seen as a prominent public health approach. Adaptable cost effective approach to community-based stroke prevention and clinical setting readiness for the management common causes of stoke is vital [17]. So it is imperative that a lot has to be done to address the issues concerning identification of stroke subtypes and stroke diagnosis in Ethiopia.

\section{Methods and Participants}

The study was conducted at stroke unit of Jimma University Medical Center (JUMC), a tertiary hospital found in Jimma city, southwest Ethiopia, which is 352 kilometers from Addis Ababa. It is the main referral center for neurology patients in south west Ethiopia. Stroke unit of JUMC was established with assistance of project from United Kingdom Aid Direct (UKAID) and Tropical Health and Education Trust (THET) with the help of Southampton hospital at the beginning of 2015. Prospective observational study design was carried out for 4 consecutive months from March 10- July 10, 2017.

The Study population were all patients $>18$ years having either clinical diagnosis or confirmed by imaging as per WHO criteria for diagnosis of stroke [18] and admitted to stroke unit of JUMC within the study period.

\section{Exclusion criteria}

- Patient or guardian not willing to give an informed consent.

- Those who died before evaluation by stroke neurologist/other physician

- After further evaluation if initial assessment or diagnosis of stroke was later changed to other case (ruled out stroke) as long as the patient was in the stroke unit.

- Patients with diagnosis of TIA and hematomas.

- Brain ischemia with hemorrhagic transformation and vice versa.

- Stroke with undetermined type clinically, where imaging unaffordable.

\section{Sample Size Determination}

The sample size was calculated using open epi version 7.0.9.34 statistical package. With Epi info version 7.0.9.34 the minimum sample size calculated was 88 patients. But in this study all eligible study participants during the study period, 116 stroke patients $(60$ ischemic stroke and 56 hemorrhagic stroke patients) were included. Eligible study participants were selected by using convenience sampling technique.

\section{Discussion}

\section{Data collection tool and procedure}

Data collection was carried out by two dedicated BSc nurses and one internal medicine resident with special training in stroke and contents of the study. Data collectors collect all relevant data from the case records and interview using a standardized data extraction form. The needed history used for the study was taken from the patient and/ or relatives in the language they understood. Controversial data was discussed with stroke physicians working in the stroke unit of JUMC during study period. The decision to perform different ancillary tests, laboratories, imaging and clinical history taking was left to the treating clinicians. Data collection tool include Sociodemographic, stroke subtypes, biochemical and laboratory profile, as well as findings of imaging's like chest X-rays, electrocardiography, echocardiography and brain imaging.

\section{Data processing and analysis}

Checked data was cleaned and entered to Epidata version 3.1 and analyzed using statistical package for the social sciences (SPSS) for windows version 20 (Chicago, USA). Descriptive statistics such as proportions, means, standard deviations, medians, and interquartile ranges were calculated to describe the sociodemographic characteristics and diagnostic protocols among stroke patients admitted to stroke unit of JUMC. Chi-square $(\chi 2)$ test was used to test the significance of associations between categorical variables and Kolmogorov-Smirnov test was used to assess the distribution of variables. Confidence interval which doesn't contain 1 and predictors with probability value less than 0.05 was considered statistically significant.

\section{Operational definition and clinical scale of the patient status}

Ischemic stroke: Evidence of a recent infarct in the clinically relevant area of the brain/confirmed cerebral infarction [4,19]. A brain CT scan showing a hypo dense area matching the clinical picture [20].

Intracerebral hemorrhage: Bleeding from one of the brain's arteries (mostly from intracerebral arteries) into the brain tissue [21].

Hemorrhagic stroke: Occurs due to the weakening of blood vessel which would rupture and bleed into the surrounding brain tissues [12].

Subarachnoid hemorrhage: A bleeding from intra cranial arteries leading to blood between two membranes that surround the brain (arterial bleeding in the space between the two meninges, piamater and arachnoid) [21].

\section{Type of Ischemic Strokes, As Toast (Trial of Org 10172 in Acute Stroke Treatment) Classification}

- Macroangiopathy/Large-Artery Atherosclerosis (LAA): The presence of an occlusion or a stenosis with $>50 \%$ diameter reduction of a brain-supplying artery corresponding to clinical symptoms and with location and morphology typical of atherosclerosis on doppler ultrasound or angiography. Diagnostic studies had to exclude potential sources of cardiogenic embolism [20].

- Microangiopathy/small-vessel disease or Lacunar Stroke (LS): The presence of one of the traditional lacunar syndromes (eg, pure motor stroke, pure sensory stroke, sensory motor stroke, ataxic hemiparesis, and dysarthria-clumsy hand syndrome), infarction(s) $<1.5 \mathrm{~cm}$ of diameter or normal CT/ MRI examination, and absence of acute cerebral cortical dysfunction. Potential cardiac sources for embolism should be absent, and evaluation of the large extra cranial arteries should not demonstrate a stenosis of $>50 \%$ in an ipsilateral artery [20].

- Cardio-Embolic Stroke (CE): The presence of a high or medium-risk source of cardiac embolism in the absence of potential LAA sources of thrombosis or embolism [20].

- Other Defined Etiologies (OD): Conditions such as spontaneous or traumatic dissection, vasculitis, fibromuscular dysplasia, hematologic disorders, coagulopathies, or other not-further 
specified diseases. These conditions had to be confirmed by angiography or blood tests. CE and LAA had to be excluded [20].

- Undetermined Etiology (UE): patients in whom a likely etiology could not be determined despite an extensive evaluation or in whom the treating physician felt that not all necessary investigations had been performed [20].

\section{Laboratory reference ranges for normal persons (JUMC laboratory center)}

Serum electrolytes

- $\quad$ Calcium (ionized):4.61-5.33 mg/dL

- $\quad$ Potassium: $3.50-5.10 \mathrm{mmol} / \mathrm{L}$

- $\quad$ Sodium: $135.0-145.0 \mathrm{mmol} / \mathrm{L}$

- Chlorine: $95.0-115.0 \mathrm{mmol} / \mathrm{L}$

- $\quad$ PH: $7.20-7.60$

Cardiac biomarkers (Vidas automated immune analyzer)

- Troponin: $<0.01 \mathrm{ng} / \mathrm{mL}$

- $\quad$ CK-MB: $<5.00 \mathrm{ng} / \mathrm{ml}$

Complete blood count (SYSMEX xs 500i)

- $\quad$ WBC: $3.00-15.00 \mathrm{X} 103 / \mu \mathrm{L}$

- $\quad$ HGB: $8.0-17.0 \mathrm{~g} / \mathrm{dL}$

- HCT: $26.0-50.0 \%$

- $\quad \mathrm{MCV}: 86.0-110.0 \mathrm{fL}$

- Platelet: $150-400 \mathrm{X} 103 / \mu \mathrm{L}$

Coagulation profile

- PT: 10-14 seconds

- $\quad$ aPTT: 22-27.9 seconds

- INR: $0.8-1.1$

Renal function test (Mindray BS 200e)

- $\quad$ Creatinine: male: $0.6-1.1 \mathrm{mg} / \mathrm{dL}$, female: $0.5-0.9 \mathrm{mg} / \mathrm{dL}$

- Urea: $15.00-43.20 \mathrm{mg} / \mathrm{dL}$

Liver function test (Mindray BS 200e)

- $\quad$ AST: male $=0-37 \mathrm{U} / \mathrm{L}$, female $=0-31 \mathrm{U} / \mathrm{L}$

- $\quad$ ALT: male $=0-42 \mathrm{U} / \mathrm{L}$, female $=0-32 \mathrm{U} / \mathrm{L}$

- ALP: $\mathrm{male}=80-306 \mathrm{U} / \mathrm{L}$, female $=64-306 \mathrm{U} / \mathrm{L}$

- Direct bilirubin: 0-0.02 mg/dL

- Total bilirubin:0.1-1.20 mg/dL

Others

- Uric acid: male=3.4-7.0 mg/dL, female $=2.4-5.7 \mathrm{mg} / \mathrm{dL}$

- TFT: TSH: $0.25-5 \mu \mathrm{IU} / \mathrm{mL}, \quad \mathrm{T} 4: \quad 60-120 \mathrm{nmol} / \mathrm{L}, \quad \mathrm{T} 3$ : $0.92-2.33 \mathrm{nmol} / \mathrm{L}$

- ESR: male: $0-20 \mathrm{~mm} / \mathrm{h}$, Female: $0-30 \mathrm{~mm} / \mathrm{h}$

\section{Results}

\section{Sociodemographic characteristics of the patients}

During the study period 116 study participants were included in the final analysis. The mean age of the patients was $55.14+14.04$ years (ranged, 23 to 96 years). Males comprised of 73 (62.9\%) with male: female ratio of 1.70:1. One hundred four patients were married and none of the patient was unmarried/single. Majority of the participants had informal education (able to read and write) 49(42.2\%) followed by no basic education (unable to write and read) $42.2 \%$. Majority of the patients were farmers $44(37.9 \%)$ followed by home housewives $41(35.3 \%)$. With this majority of them were unemployed being doing their own/self-work 101 (87.1\%) (Table 1).

\section{Approaches of stroke diagnosis and stroke sub-types}

Of the 116 patients, 61 patients (52.6\%) had CT scan of the brain performed, whereas $55(47.4 \%)$ of patients were evaluated clinically alone to have stroke. Using WHO criteria, 60 (51.7\%) patients had ischemic stroke while 56 (48.3\%) had hemorrhagic, with intracerebral hemorrhage ( $\mathrm{ICH})$ and subarachnoid hemorrhage (SAH) accounting for $44.0 \%$ and $4.3 \%$, respectively. According to the CT scan findings, 30 $(49.2 \%)$ patients were found to have infarction while $31(50.8 \%)$ had hemorrhagic stroke (Table 2).

The median time to perform CT scan after hospital arrival was 4 days (ranged: 0.13-17.13 days). From the 61 patients with stroke confirmed by CT scan, $34.4 \%$ were scanned within 3 days of hospital arrival, $50.8 \%$ between 3 days and 7 days and $13.1 \%$ between 7 days and 14 days. Only 1 patient had CT scan 2 weeks after the hospital arrival. From those patients who had brain imaging the result complied with clinical diagnosis in 48 (78.7\%) patients (90\% of IS and $67.7 \%$ of HS)

Etiologic investigation for stroke was infrequently performed: 37 (31.9\%) of the patients had an electrocardiogram, 12 (10.3\%) had an echocardiogram, 11 patients had chest X-ray and 2 (1.7\%) had Doppler ultrasound. From 37 patients for whom ECG was done; 20 (54.1\%) had left ventricular hypertrophy and 17 (45.9\%) had atrial fibrillation. For 12 patients with etiologic evaluation with echocardiogram; $(41.7 \%)$ had left ventricular hypertrophy and $6(50.0 \%)$ had low ejection fraction. Rheumatic valvular heart diseases was confirmed in one young stroke patient.

\section{Topographic distribution of stroke and sites of brain involvement}

The most common site of lesion/altered areas of brain on CT scan of hemorrhage stroke patients was basal ganglia (48.4\%) followed by parietal lobe (29.0\%), ventricular (29.0\%), thalamus (25.8\%) and frontal lobe $(22.6 \%)$. The most common site of brain infarct was parietal lobe (33.3\%) and cortical (33.3\%), followed by basal ganglia (23.3\%), caudate nucleus (20.0\%), and temporal lobe (16.7\%) (Table 2).

Based on CT scan findings, among 30 ischemic stroke cases confirmed by CT scan, 8 (26.7\%) had right hemisphere involvement, 7 (23.3\%) had left hemisphere involvement, 7 (23.3\%) had anterior circulation involvement, 2 (6.7\%) had massive cerebral infarction involvement and $2(6.7 \%)$ had posterior circulation involvement. Up on CT scan of hemorrhagic patients, $19(61.3 \%)$ had intraparenchymal bleed (IPB) only, 8 (25.8\%) intraparenchymal bleed with interventricular extension, 2 (6.5\%) subarachnoid bleed (SAB) only, 1 (3.2\%) intraparenchymal bleed with subarachnoid extension and 1 (3.2\%) had subarachnoid bleed with interventricular involvement. 
Citation: Fekadu G, Chelkeba L, Melaku T, Tegene E (2018) Pathological Sub Types and Diagnostic Protocols of Stroke among Adult Patients Admitted to Jimma University Medical Center, South West Ethiopia. J Neurol Neurophysiol 9: 466. doi:10.4172/2155-9562.1000466

Page 4 of 18

\begin{tabular}{|c|c|c|c|c|c|}
\hline \multicolumn{2}{|c|}{ Sociodemographic and other patient related factors } & \multirow{2}{*}{$\begin{array}{c}\begin{array}{c}\text { Total patients } \\
(\mathbf{n}=116)\end{array} \\
55.14+14.04 \\
\end{array}$} & \multirow{2}{*}{$\begin{array}{c}\text { Ischemic stroke }(\mathbf{n}=\mathbf{6 0}) \\
57.38+14.59\end{array}$} & \multirow{2}{*}{$\begin{array}{c}\text { Hemorrhagic stroke } \\
(\mathbf{n = 5 6 )}\end{array}$} & \multirow{2}{*}{$\begin{array}{c}\text { X2 (P value) } \\
0.187\end{array}$} \\
\hline Age (years) & Mean \pm SD & & & & \\
\hline \multirow{2}{*}{ Sex } & Male & $73(62.9 \%)$ & $38(63.3 \%$ & $35(62.5 \%$ & 0.926 \\
\hline & Female & $43(37.1 \%)$ & $22(36.7 \%)$ & $21(37.5 \%)$ & \\
\hline \multirow{2}{*}{ Residence } & Rural & $84(72.4 \%)$ & $43(71.7 \%)$ & $41(73.2 \%)$ & 0.852 \\
\hline & Urban & $32(27.6 \%$ & $17(28.3 \%)$ & $15(26.8 \%)$ & \\
\hline \multirow{3}{*}{ Marital status } & Married & $104(89.7 \%)$ & $54(90.0 \%)$ & $50(89.3 \%)$ & 0.575 \\
\hline & Widow & $11(9.5 \%)$ & $5(8.3 \%)$ & $6(10.7 \%)$ & \\
\hline & Divorced & $1(0.9 \%)$ & $1(1.7 \%)$ & $0(0 \%)$ & \\
\hline \multirow{5}{*}{ Ethnicity } & Oromo & $82(70.7 \%)$ & $46(76.7 \%)$ & $36(64.3 \%)$ & 0.190 \\
\hline & Kafa & $15(12.9 \%)$ & $7(11.7 \%)$ & $8(14.3 \%)$ & \\
\hline & Amhara & $8(6.9 \%)$ & $5(8.3 \%)$ & $3(5.4 \%)$ & \\
\hline & Dawuro & $6(5.2 \%)$ & $1(1.7 \%)$ & $5(8.9 \%)$ & \\
\hline & Others & $5(4.3 \%$ & $1(1.7 \%)$ & $4(7.1 \%)$ & \\
\hline \multirow{4}{*}{ Religion } & Muslim & $71(61.2 \%)$ & $40(66.7 \%)$ & $31(55.4 \%$ & 0.418 \\
\hline & Orthodox & $35(30.2 \%)$ & $15(25.0 \%)$ & $20(35.7 \%)$ & \\
\hline & Protestant & $9(7.8 \%)$ & $4(6.7 \%)$ & $5(8.9 \%)$ & \\
\hline & Traditional belief & $1(0.9 \%)$ & $1(1.7 \%)$ & $0(0 \%)$ & \\
\hline \multirow{5}{*}{ Education status } & Unable to read and write & $42(36.2 \%)$ & $20(33.3 \%)$ & $22(39.3 \%)$ & 0.459 \\
\hline & Able to read and write, informal education & $49(42.2 \%)$ & $28(46.7 \%)$ & $21(37.5 \%)$ & \\
\hline & Elementary school (1-8) & $17(14.7 \%)$ & $10(16.7 \%)$ & $7(12.5 \%)$ & \\
\hline & Secondary school (9-12) & $3(2.6 \%)$ & $1(1.7 \%)$ & $2(3.6 \%)$ & \\
\hline & College/university or above & $5(4.3 \%)$ & $1(1.7 \%)$ & $4(7.1 \%)$ & \\
\hline \multirow{7}{*}{$\begin{array}{l}\text { Occupational status (over the } \\
\text { last 1years) }\end{array}$} & Agriculture / farmer & $44(37.9 \%)$ & $27(45.0 \%)$ & $17(30.4 \%)$ & 0.354 \\
\hline & Homemaker/ housewives & $41(35.3 \%$ & $20(33.3 \%)$ & $21(37.5 \%)$ & \\
\hline & Merchant & $11(9.5 \%)$ & $7(11.7)$ & $4(7.1 \%)$ & \\
\hline & Retired & $6(5.2 \%$ & $2(3.3 \%)$ & $4(7.1 \%)$ & \\
\hline & Government employee & $5(4.3 \%)$ & $1(1.7 \%)$ & $4(7.1 \%)$ & \\
\hline & Other own business work & $5(4.3 \%)$ & $2(3.3 \%)$ & $3(5.4 \%)$ & \\
\hline & Skilled/unskilled manual labor/ daily worker & $4(3.4 \%)$ & $1(1.7 \%)$ & $3(5.4 \%)$ & \\
\hline
\end{tabular}

Table 1: Sociodemographic characteristics of stroke among adult patients admitted to stroke unit of JUMC from March 10-July 10, 2017 ( ${ }^{\star}$ Ethnicity others: Silte, Yem, Tigire Nuwer: SD: Standard Deviation).

\section{Etiology of ischemic strokes, according to the TOAST criteria}

The predominant stroke etiology was undetermined etiology in 26 patients $(43.3 \%)$, followed by cardioembolism in 22 patients $(36.7 \%)$, large artery atherosclerosis (LAA) in 7 patients (11.7\%) and small vessel occlusion (SVO) in 5 patients (8.3\%), of all ischemic stroke patients.

\section{Laboratory data/biochemical parameters of the patients}

During the study period $115(99.1 \%)$ patients' blood glucose, $114(98.3 \%)$ renal function test, 113 (97.4\%) sero-status, $112(96.6 \%)$ complete blood count, $96(82.8 \%)$ liver function test, 58 (50.0\%) coagulation profile, 50 (43.1) lipid panel test, 52 (44.8\%) cardiac biomarker and 46 (39.7\%) serum electrolyte was done. Other preliminary laboratory tests done were erythrocyte sedimentation rate (ESR), thyroid function test and uric acid level. The laboratory values considered was only the initial laboratory value (one shot) during hospital stay.

Regarding serum electrolyte, majority of the patients had normal electrolyte level of potassium, sodium, chlorine and $\mathrm{PH}$ level ranging from $54.3 \%$ to $91.3 \%$, but $69.6 \%$ of the patients had hypocalcaemia. The low calcium level had no significant difference between stroke types $(\mathrm{P}=0.109)$. The median level of lipid panel profile was almost in optimal range, $176.4 \mathrm{mg} / \mathrm{dL}$ for TC, $45.55 \mathrm{mg} / \mathrm{dL}$ for $\mathrm{HDL}, 108.5 \mathrm{mg} / \mathrm{dL}$ for LDL-C and $112 \mathrm{mg} / \mathrm{dL}$ for TG.

Renal function test, CBC, coagulation tests, cardiac biomarkers, liver function tests and ESR in majority of the patients was within normal range with no significant difference in stroke type. Hemoglobin $<11 \mathrm{~g} / \mathrm{dL}$, leukocytosis ( $>15000$ cells $/ \mathrm{mm}^{3}$ ) and thrombocytopenia $\left(<150000\right.$ cells $\left./ \mathrm{mm}^{3}\right)$ were found in $6.3 \%, 3.6 \%$ and $4.5 \%$ of patients respectively. The median hemoglobin and random blood sugar level was $14.55 \mathrm{~g} / \mathrm{dL}$ (ranged:2.2-25.1 g/dL) and $115 \mathrm{mg} / \mathrm{dL}$ (ranged: 60$600 \mathrm{mg} / \mathrm{dL}$ ) respectively, with no significant difference between stroke type. Hyperglycemia (random blood sugar $>200 \mathrm{mg} / \mathrm{dL}$ ) and deranged renal function (creatinine $>1.1 \mathrm{mg} / \mathrm{dL}$ ) occurred in $6.1 \%$ and $16.7 \%$ respectively (Table 3 ).

\section{Discussion}

Of the total patients, $52.6 \%$ of the patients had CT scan of the brain performed, whereas the remaining patients were evaluated clinically alone to have stroke. This CT scan performance rate was higher than study conducted in northern Ethiopia by Greffie et al. which was $38.5 \%$ [17], and lower than other study in other northern part of Ethiopia by Gebremariam et al. 76.1\% [13], Tirschwell et al. in Vietnam 99.6\% [22] and De Carvalho et al. in Brazil in 92.9\% of the patients [23]. The difference in CT scan performance rate in different setting was associated with the functionality, availability and affordability by the patient. In our set up because of frequent malfunctioning, shortage of expert and cost issue about half of the patients were diagnosed clinically alone. Clinical diagnosis alone may not reflect the true nature of stroke in our patients. In addition to the affordability, only one CT scan device 
Citation: Fekadu G, Chelkeba L, Melaku T, Tegene E (2018) Pathological Sub Types and Diagnostic Protocols of Stroke among Adult Patients Admitted to Jimma University Medical Center, South West Ethiopia. J Neurol Neurophysiol 9: 466. doi:10.4172/2155-9562.1000466

Page 5 of 18

\begin{tabular}{|c|c|c|c|c|}
\hline \multicolumn{2}{|l|}{ Stroke diagnosis } & \multirow{2}{*}{$\begin{array}{c}\text { Total patients } \\
\quad(\mathrm{N}=116)\end{array}$} & \multirow{2}{*}{$\begin{array}{c}\text { Ischemic stroke } \\
(n=60)\end{array}$} & \multirow{2}{*}{$\begin{array}{c}\text { Hemorrhagic stroke } \\
(n=56)\end{array}$} \\
\hline \multirow{2}{*}{ Way of stroke diagnosis } & Clinical only & & & \\
\hline & Brain imaging / CT-scan & $61(52.6 \%)$ & $30(50.0 \%)$ & $31(55.4 \%)$ \\
\hline \multicolumn{2}{|l|}{ Electrocardiogram (ECG) } & $37(31.9 \%)$ & $25(41.7 \%)$ & $12(21.4 \%)$ \\
\hline & Left ventricular hypertrophy & $20(54.1 \%)$ & $14(56.0 \%)$ & $6(50.0 \%)$ \\
\hline & Atrial fibrillation & $17(45.9 \%)$ & $13(52.0 \%)$ & $4(33.3 \%)$ \\
\hline & Sinus tachycardia & $5(13.5 \%)$ & $3(12.0 \%)$ & $2(16.7 \%)$ \\
\hline & Ischemic heart disease & $2(5.4 \%)$ & $2(8.0 \%)$ & $0(0 \%)$ \\
\hline & Sinus bradycardia & $2(5.4 \%)$ & $1(4.0 \%)$ & $1(8.3 \%)$ \\
\hline & Normal finding. & $7(18.9 \%)$ & $5(20.0 \%)$ & $2(16.7 \%)$ \\
\hline \multicolumn{2}{|c|}{ Echocardiogram (trans esophageal, transthoracic, or both) } & $12(10.3 \%)$ & $11(18.3 \%)$ & $1(1.8 \%)$ \\
\hline & Low EF & $6(50.0 \%)$ & $5(45.5 \%)$ & $1(100 \%)$ \\
\hline & Left ventricular hypertrophy & $5(41.7 \%)$ & $4(36.4 \%)$ & $1(100 \%)$ \\
\hline & Dilated cardiomyopathy & $3(25.0 \%)$ & $3(27.3 \%)$ & $0(0 \%)$ \\
\hline & Ischemic cardiomyopathy with severe systolic dysfunction & $3(25.0 \%)$ & $3(27.3 \%)$ & $0(0 \%)$ \\
\hline & Rheumatic Valvular heart disease & $1(8.3 \%)$ & $1(9.1 \%)$ & $0(0 \%)$ \\
\hline & Ischemic heart disease with mural thrombus/LV apical thrombus & $1(8.3 \%)$ & $1(9.1 \%)$ & $0(0 \%)$ \\
\hline & Ventricular wall motion abnormalities & $1(8.3 \%)$ & $1(9.1 \%)$ & $0(0 \%)$ \\
\hline & Normal & $1(8.3 \%)$ & $1(9.1 \%)$ & $0(0 \%)$ \\
\hline \multirow{2}{*}{$\begin{array}{l}\text { Doppler US } \\
(n=2)\end{array}$} & Atherosclerosis without stenosis & $1(0.9 \%)$ & $0(0 \%)$ & $1(50.0 \%)$ \\
\hline & Normal finding & $1(0.9 \%)$ & $0(0 \%)$ & $1(50.0 \%)$ \\
\hline \multirow{15}{*}{$\begin{array}{l}\text { Lesion site/ altered areas of } \\
\text { brain on CT scan }(n=61)\end{array}$} & Basal ganglia & $22(36.1 \%)$ & $7(23.3 \%)$ & $15(48.4 \%)$ \\
\hline & Parietal lobe & $19(31.1 \%)$ & $10(33.3 \%)$ & $9(29.0 \%)$ \\
\hline & Cortical & $14(23.0 \%)$ & $10(33.3 \%)$ & $4(12.9 \%)$ \\
\hline & Thalamus & $13(21.3 \%)$ & $5(16.7 \%)$ & $8(25.8 \%)$ \\
\hline & Frontal lobe & $11(18.0 \%)$ & $4(13.3 \%)$ & $7(22.6 \%)$ \\
\hline & Cerebellar & $10(16.4 \%)$ & $4(13.3 \%)$ & $6(19.4 \%)$ \\
\hline & Ventricular & $9(14.8 \%)$ & $0(0 \%)$ & $9(29.0 \%)$ \\
\hline & Caudate nucleus & $8(13.1 \%)$ & $6(20.0 \%)$ & $2(6.5 \%)$ \\
\hline & Occipital lobe & $6(9.8 \%)$ & $4(13.3 \%)$ & $2(6.5 \%)$ \\
\hline & Temporal lobe & $5(8.2 \%)$ & $5(16.7 \%)$ & $1(3.2 \%)$ \\
\hline & Internal capsule & $3(4.9 \%)$ & $2(6.7 \%)$ & $1(3.2 \%)$ \\
\hline & Brainstem & $3(4.9 \%)$ & $1(3.3 \%)$ & $2(6.5 \%)$ \\
\hline & Insula lobe & $2(3.3 \%)$ & $2(6.7 \%)$ & $0(0 \%)$ \\
\hline & Lentiform nucleus & $2(3.3 \%)$ & $2(6.7 \%)$ & $0(0 \%)$ \\
\hline & Midbrain & $1(1.6 \%)$ & $0(0 \%)$ & $1(3.2 \%)$ \\
\hline
\end{tabular}

Table 2: Approaches of stroke diagnosis and stroke types among adult patients admitted to stroke unit of JUMC from March 10-July $10,2017$.

\begin{tabular}{|c|c|c|c|c|c|}
\hline \multicolumn{3}{|l|}{ Laboratory parameters } & \multirow{2}{*}{$\begin{array}{l}\text { Total patients } \\
(n=116)\end{array}$} & \multirow{2}{*}{$\begin{array}{c}\text { Ischemic stroke } \\
(n=60)\end{array}$} & \multirow{2}{*}{$\begin{array}{c}\text { Hemorrhagic stroke } \\
(n=56)\end{array}$} \\
\hline \multirow{4}{*}{$\begin{array}{l}\text { Renal function tests } \\
(n=114)\end{array}$} & \multirow{2}{*}{ Creatinine } & Normal & & & \\
\hline & & Elevated & $19(16.7 \%)$ & $12(20.7 \%)$ & $7(12.5 \%)$ \\
\hline & \multirow{2}{*}{$\begin{array}{l}\text { BUN or Urea } \\
(N=113)\end{array}$} & Normal & $85(75.2 \%)$ & $44(77.2 \%)$ & $41(73.2 \%)$ \\
\hline & & Elevated & $28(24.8 \%)$ & $13(22.2 \%$ & $15(26.8 \%)$ \\
\hline \multirow{11}{*}{$\begin{array}{l}\text { Complete blood count with } \\
\text { differential cell count } \\
(n=112)\end{array}$} & \multirow{3}{*}{ White blood cells/microliter } & Leukopenia & $3(2.7 \%)$ & $2(3.4 \%)$ & $1(1.9 \%)$ \\
\hline & & Normal WBC & $105(93.8 \%)$ & $54(93.1 \%)$ & $51(94.4 \%)$ \\
\hline & & Leukocytosis & $4(3.6 \%)$ & $2(3.4 \%)$ & $2(3.7 \%)$ \\
\hline & \multirow{3}{*}{ Hemoglobin (g/dL) } & $<11 \mathrm{~g} / \mathrm{dl}$ & $7(6.3 \%)$ & $4(6.9 \%)$ & $3(5.6 \%)$ \\
\hline & & $11-16 \mathrm{~g} / \mathrm{dl}$ & $86(76.8 \%)$ & $44(75.9 \%)$ & $42(77.8 \%)$ \\
\hline & & $>16 \mathrm{~g} / \mathrm{dl}$ & $19(17.0 \%)$ & $10(17.2 \%)$ & $9(16.7 \%)$ \\
\hline & \multirow[t]{2}{*}{$\mathrm{MCV}(\mathrm{fl})$} & Microcytic anemia & $4(3.6 \%)$ & $3(5.2)$ & $1(1.9 \%)$ \\
\hline & & Normocytic anemia & $108(96.4 \%)$ & $55(94.8 \%)$ & $53(98.1 \%)$ \\
\hline & \multirow{3}{*}{ Platelet counts } & Thrombocytopenia & $5(4.5 \%)$ & $3(5.2 \%)$ & $2(3.7 \%)$ \\
\hline & & Normal & $106(94.6 \%)$ & $54(93.1 \%)$ & $52(96.3 \%)$ \\
\hline & & Thrombocytosis & $1(0.9 \%)$ & $1(1.7 \%)$ & $0(0 \%)$ \\
\hline
\end{tabular}


Citation: Fekadu G, Chelkeba L, Melaku T, Tegene E (2018) Pathological Sub Types and Diagnostic Protocols of Stroke among Adult Patients Admitted to Jimma University Medical Center, South West Ethiopia. J Neurol Neurophysiol 9: 466. doi:10.4172/2155-9562.1000466

Page 6 of 18

\begin{tabular}{|c|c|c|c|c|c|}
\hline \multirow{9}{*}{$\begin{array}{l}\text { Liver function tests } \\
(n=96)\end{array}$} & \multirow{2}{*}{$\begin{array}{l}\text { AST } \\
(n=95)\end{array}$} & Normal & $72(75.8 \%)$ & $38(77.6 \%)$ & $34(73.9 \%)$ \\
\hline & & elevated & $23(24.2 \%)$ & $11(22.4 \%)$ & $12(26.1 \%)$ \\
\hline & \multirow{2}{*}{$\begin{array}{l}\text { ALT } \\
(n=95)\end{array}$} & Normal & $79(83.2 \%)$ & $39(79.6 \%)$ & $40(87.0 \%)$ \\
\hline & & elevated & $16(16.8 \%)$ & $10(20.4 \%)$ & $6(13.0 \%)$ \\
\hline & \multirow{2}{*}{$\begin{array}{l}\text { ALP } \\
(n=26)\end{array}$} & Normal & $13(50.0 \%)$ & $8(50.0 \%)$ & $5(50.0 \%)$ \\
\hline & & elevated & $13(50.0 \%)$ & $8(50.0 \%)$ & $5(50.0 \%)$ \\
\hline & \multirow{2}{*}{$\begin{array}{l}\text { Albumin } \\
(n=3)\end{array}$} & Low & $1(33.3 \%)$ & $1(33.3 \%)$ & $0(0 \%)$ \\
\hline & & Normal & $2(67.7 \%)$ & $2(67.7 \%)$ & $0(0 \%)$ \\
\hline & Bilirubin(n=3) & Normal & $3(60.0 \%)$ & $2(50.0 \%)$ & $1(100 \%)$ \\
\hline \multirow{6}{*}{$\begin{array}{l}\text { Coagulation tests } \\
(n=58)\end{array}$} & \multirow{2}{*}{$\begin{array}{l}\operatorname{aPTT}(\mathrm{sec}) \\
(\mathrm{n}=43)\end{array}$} & Normal & $28(65.1 \%)$ & $13(54.2 \%)$ & $15(78.9 \%)$ \\
\hline & & Elevated & $15(34.9 \%)$ & $11(45.8 \%)$ & $4(21.1 \%)$ \\
\hline & \multirow{2}{*}{$\begin{array}{l}\text { PT (sec) } \\
(n=42)\end{array}$} & Normal & $23(54.8 \%)$ & $11(47.8 \%)$ & $12(63.2 \%)$ \\
\hline & & Elevated & $19(45.2 \%)$ & $12(52.2 \%)$ & $7(36.8 \%)$ \\
\hline & \multirow{2}{*}{$\begin{array}{l}\text { INR } \\
(n=57)\end{array}$} & Normal & $24(42.1 \%)$ & $12(37.5 \%)$ & $12(58.0 \%)$ \\
\hline & & Elevated & $33(57.9 \%)$ & $20(62.5 \%)$ & $13(52.0 \%)$ \\
\hline \multirow{4}{*}{$\begin{array}{l}\text { Cardiac biomarkers } \\
(n=52)\end{array}$} & \multirow{2}{*}{$\begin{array}{l}\text { Troponin } \\
(n=52)\end{array}$} & Normal & $40(76.9 \%)$ & $21(67.7 \%)$ & $19(90.5 \%)$ \\
\hline & & Elevated & $12(23.1 \%)$ & $10(32.3 \%)$ & $2(9.5 \%)$ \\
\hline & \multirow{2}{*}{$\begin{array}{l}\text { CK.MB } \\
(n=12)\end{array}$} & Normal & $8(66.7 \%)$ & $2(40.0 \%)$ & $6(85.7 \%)$ \\
\hline & & Elevated & $4(33.3 \%)$ & $3(60.0 \%)$ & $1(14.3 \%)$ \\
\hline \multirow{13}{*}{$\begin{array}{l}\text { Lipid profile tests } \\
(n=50)\end{array}$} & \multirow{3}{*}{ Total cholesterol (TC) } & $<200 \mathrm{mg} / \mathrm{dl}$ & $33(66.0 \%)$ & $22(71.0 \%)$ & $11(57.9 \%)$ \\
\hline & & $200-239.9 \mathrm{mg} / \mathrm{dl}$ & $12(24.0 \%)$ & $6(19.4 \%)$ & $6(31.6 \%)$ \\
\hline & & $\geq 240 \mathrm{mg} / \mathrm{dl}$ & $5(10.0 \%)$ & $3(19.4 \%)$ & $2(10.6 \%)$ \\
\hline & \multirow{3}{*}{ HDL-C } & $<40 \mathrm{mg} / \mathrm{dl}$ & $19(38 \%)$ & $13(41.9 \%)$ & $6(31.6 \%)$ \\
\hline & & $40-59.9 \mathrm{mg} / \mathrm{dl}$ & $24(48.0 \%)$ & $15(48.4 \%)$ & $9(47.4 \%)$ \\
\hline & & $\geq 60 \mathrm{mg} / \mathrm{dl}$ & $7(14.0 \%)$ & $3(9.7 \%)$ & $4(21.1 \%)$ \\
\hline & \multirow{4}{*}{ LDL-C } & $<100 \mathrm{mg} / \mathrm{dl}$ & $22(44.0 \%)$ & $12(38.7 \%)$ & $10(52.6 \%)$ \\
\hline & & $100-129.9 \mathrm{mg} / \mathrm{dl}$ & $14(28.0 \%)$ & $9(29.0 \%)$ & $5(26.3 \%)$ \\
\hline & & $130-159.9 \mathrm{mg} / \mathrm{dl}$ & $8(16.0 \%)$ & $7(22.6 \%)$ & $1(5.3 \%)$ \\
\hline & & $\geq 160 \mathrm{mg} / \mathrm{dl}$ & $6(12.0 \%)$ & $3(9.7 \%)$ & $3(15.8 \%)$ \\
\hline & \multirow{3}{*}{ Triglyceride (TG) } & $<150 \mathrm{mg} / \mathrm{dl}$ & $40(80.0 \%)$ & $24(77.4 \%)$ & $16(84.2 \%)$ \\
\hline & & $150-199.9 \mathrm{mg} / \mathrm{dl}$ & $8(16.0 \%)$ & $6(19.4 \%)$ & $2(10.5 \%)$ \\
\hline & & $\geq 200$ & $2(4.0 \%)$ & $1(3.2 \%)$ & $1(5.3 \%)$ \\
\hline \multirow{15}{*}{$\begin{array}{l}\text { Serum electrolytes done } \\
(n=46)\end{array}$} & & Low & $32(69.6 \%)$ & $14(60.9 \%)$ & $18(78.3 \%)$ \\
\hline & Calcium & Normal & $13(28.3 \%)$ & $9(39.1 \%)$ & $4(17.4 \%)$ \\
\hline & & Elevated & $1(2.2 \%)$ & $0(0 \%)$ & $1(4.3 \%)$ \\
\hline & & Low & $4(8.7 \%)$ & $1(4.3 \%)$ & $3(13.0 \%)$ \\
\hline & Potassium & Normal & $41(89.1 \%)$ & $21(91.3 \%)$ & $20(87.0 \%)$ \\
\hline & & Elevated & $1(2.2 \%)$ & $1(4.3 \%)$ & $0(0 \%)$ \\
\hline & & Low & $14(30.4 \%)$ & $4(17.4 \%)$ & $10(43.5 \%)$ \\
\hline & Sodium & Normal & $30(54.3 \%)$ & $17(73.9 \%)$ & $13(56.5 \%)$ \\
\hline & & Elevated & $2(4.3 \%)$ & $2(8.7 \%)$ & $0(0 \%)$ \\
\hline & & Low & $3(6.5 \%)$ & $1(4.3 \%)$ & $2(8.7 \%)$ \\
\hline & Chlorine & Normal & $42(91.3 \%)$ & $22(95.7 \%)$ & $20(87.0 \%)$ \\
\hline & & Elevated & $1(2.2 \%)$ & $0(0 \%)$ & $1(4.3 \%)$ \\
\hline & & Low & $3(6.5 \%)$ & $1(4.3 \%)$ & $2(8.7 \%)$ \\
\hline & $\mathrm{PH}$ & Normal & $29(63.0 \%)$ & $16(69.6 \%)$ & $13(56.5 \%)$ \\
\hline & & Elevated & $14(30.42 \%)$ & $6(26.1 \%)$ & $8(34.8 \%)$ \\
\hline & RBS & $<200 \mathrm{mg} / \mathrm{dl}$ & $108(93.9 \%)$ & $56(94.9 \%)$ & $52(92.9 \%)$ \\
\hline & $(n=115)$ & $\geq 200 \mathrm{mg} / \mathrm{dl}$ & $7(6.1 \%)$ & $3(5.1 \%)$ & $4(7.1 \%)$ \\
\hline & Sero status & Reactive & $1(0.9 \%)$ & $1(1.7 \%)$ & $0(0 \%)$ \\
\hline & $(n=113)$ & Non-reactive & $112(99.1 \%)$ & $58(98.3 \%)$ & $54(100 \%)$ \\
\hline & ESR & Normal & $8(80.0 \%)$ & $2(50.0 \%)$ & $6(100 \%)$ \\
\hline Others & $(n=10)$ & Elevated & $2(20.0 \%)$ & $2(50.0 \%)$ & $0(0 \%)$ \\
\hline & Uric acid & Normal & $1(50.0 \%)$ & $0(0 \%)$ & $1(100 \%)$ \\
\hline & $(n=2)$ & Elevated & $1(50.0 \%)$ & $1(100 \%)$ & $0(0 \%)$ \\
\hline & & Normal & $1(50.0 \%$ & $0(0 \%)$ & $1(100 \%)$ \\
\hline & TFT (T3 and T4) $(n=2$ ) & Elevated & $1(50.0 \%)$ & $1(100 \%)$ & $0(0 \%)$ \\
\hline
\end{tabular}

Table 3: Laboratory data/biochemical parameters of stroke among adult patients admitted to stroke unit of JUMC from March 10-July 10, 2017 (*ALP: alkaline Phosphatase, aPTT: activated Partial Thromboplastin Time, ALT: Alanine Amino Transferase, AST: Aspartate Amino Transferase, BUN: Blood Urea Nitrogen, CBC: Complete Blood Count, CK-MB: Creatinine Kinase-MB, ESR: Erythrocyte Sedimentation Rate, HDL: High Density Lipoprotein, INR: Internationalized Normal Ratio, LDL: Low Density Lipoprotein MCV: Mean Corpuscular Volume, PT: Prothrombin Time, RBS: Random Blood Sugar, TFT: Thyroid Function Test, TG: Triglyceride). 
is available for the patients in the catchment area of the hospital, which is reflected in the lack of imaging confirmation and the delay in the evaluation of the remaining patients.

Using WHO criteria, $51.7 \%$ patients had ischemic stroke while 48.3\% had hemorrhagic stroke. This was almost similar to other studies in which almost equal prevalence of stroke types were detected by Nkoku et al. in Nigeria, Tirschwell et al. in vietnam and Deresse et al. in Ethiopia $[14,22,24]$. But it was unlike to other studies where ischemic strokes were more diagnosed than hemorrhagic stroke $[3,8,12,13,17,25$ 29] ranging from $55.6-94 \%$. Additionally it was unlike to other studies where hemorrhagic stroke was more diagnosed ranging from 55.3$59.9 \%[2,30]$.

Even though ischemic stroke is still the common form of stroke, like sub-Saharan Africa hemorrhagic stroke is relatively more dominant than in other western parts of the world. This difference could be as a result of differences in study design, study setting, environmental, dietary, genetic interactions, hospital admission bias, population pyramid, socio-economic and risk factor profiles between the different populations. In some Ethiopian culture, stroke is attributed to a supernatural force requiring intervention by a traditional healer (at least in the first days of symptom onset), although individuals with severe symptoms are expected to seek medical care regardless of their cultural beliefs or ability to recognize the stroke event. These local customs may bias our sample, to an extent that is difficult to predict, toward the underestimation of less severe ischemic and overestimation of the most severe hemorrhagic strokes.

The median time to perform CT scan after hospital arrival was 4 days, similar to study by Bennour et al. in Libya mean of $3.5 \pm 2.3$ days [31] and median of 3.4 hours by De Carvalho et al. in Brazil [23]. It was delayed contrary to study by Gebremariam et al. at northern Ethiopia which was $1.6 \pm 4.7$ days [13]. From the 61 patients with stroke confirmed by CT scan, $34.4 \%$ were scanned within 3 days of hospital arrival, this delay in time of imaging was unlike to study by Sagui et al. in Senegal in which $82 \%$ of patients were got CT scan within $72 \mathrm{~h} \mathrm{[32].}$

Whether patient arrived in the appropriate time window or came late, delayed performance of brain scan is an obstacle for reperfusion therapy with IV rTPA. In our working area, whether patients come in time or delayed from symptom onset, the treatment option of treating acute ischemic stroke is only restricted to the use of antiplatelet. From those who had brain imaging, the imaging result complied with clinical diagnosis in 48 (78.7\%) stroke patients. The incorrect diagnosis caused significant effect on the management approaches in which patients with hemorrhagic strokes received antiplatelets because of wrong clinical diagnosis of hemorrhagic stroke.

Etiologic investigation for stroke was infrequently performed by the lack of systematic cardiological examinations and brain imaging's, most of the time for financial reasons and unavailability of the instruments. From 37 patients for whom ECG was done; 17 (45.9\%) had atrial fibrillation and $20(54.1 \%)$ had left ventricular hypertrophy. This was lower unlike to study by Sarkar et al. where ECG was done for 316 patients; in majority of the patients 131 (41.46\%) had LVH and had normal finding 105 (33.28\%) [30].

In this set up, even simple and inexpensive diagnostic tests like electrocardiograms were not routinely performed. It was done only for 12 patients, this was contrary to other studies where echocardiogram was done for the majority of the patients for the better diagnosis of cardiac diseases ranging from $32.3-52.8 \%$ of patients [14,23]. The presence of carotid stenosis and the presence of cardiac or aortic embolic sources were not appropriately excluded in most of our patients. Carotid Doppler ultrasound studies were done only for 2 patients unlike study by Deresse et al. $41.5 \%$ of ischemic stroke had bilateral carotid Doppler study done [14] because of poor set up for accessibility and lack of skilled personnel in our set make lesser amount of patients were benefited from this investigation.

Most common site of lesion on CT scan of hemorrhagic stroke was basal ganglia (48.4\%), similar to study by Deresse et al. in Ethiopia [14]. Unlike to this, the most common site of lesion of hemorrhagic stroke was thalamus by Vaidya CV et al. [25] and Patne et al. [3] and periventricular white matter by Kuriakose et al. [12]. The most common site of brain infarct was parietal (33.3\%) and cortical lobe (33.3\%) this was in concordant with study by Vaidya CV et al. [25], Patne et al. [3], Kuriakose et al. [12] and Ogengo et al. [33] in which parietal lobe predominates the brain infracts. Anatomical localization of the brain lesion influences prognosis, risk of recurrence, and clinical assessment and treatment decisions of the stroke.

From 30 ischemic stroke cases confirmed by CT scan, 8 (26.7\%) had right hemisphere involvement, this contrary to study by Jowi et al. in which majority of ischemic patients had anterior circulation involvement [34]. In addition majority of hemorrhagic stroke patients, 19 (61.3\%) had intraparenchymal bleed (IPB) only similar to study by Owolabi et al. [8] but unlike to study by Sarkar et al., where majority of hemorrhagic stroke patients had parenchymal hemorrhage with ventricular extension [30]. This difference could affect the treatment approaches and prognosis of the disease.

As to TOAST (Trial of Org 10172 in Acute Stroke Treatment) classification the predominant ischemic stroke etiology was undetermined etiology/cryptogenic similar to other studies $[8,32]$ mainly because of incomplete evaluation. This was unlike other study by Tan et al. in which the predominant stroke subtypes was large artery atherosclerosis (LAA) [35]. It would, however, be prudent to do a prospective study so that strict detailed clinical evaluation coupled with neuro-imaging/carotid Doppler findings would result in an accurate TOAST classification of sub-types of acute ischemic stroke.

\section{Strengths of the study}

This study looked in to the various factors associated to stroke with a continuous clinical follow-up; and provides baseline which may shed some light on the need for preventive strategy and to improve patient care and management. The major strength of this study was its prospective study design and the enrollment of consecutive patients. To ensure a uniform data collection, we ascertained consistently ascertainable diagnosis and obtained reliable information on the important ones.

\section{Limitations of the study}

The study was also associated with limitations. First, this was a hospital-based study rather than population based, hence may be subjected to referral bias. Thus extrapolations to the rest of the community should be done with caution. But prompt imaging and comprehensive investigation would not have been feasible for most patients if it were community-based.

About half the patients in this study was diagnosed clinically alone without confirmed with brain imaging which may distort the accuracy and reliability of the data to some extent. Finally, we did not explore genetic markers for stroke type. Genomic epidemiologic studies require expensive investigations of thousands of samples in the exploratory phase which is beyond the scope of this study. 
Citation: Fekadu G, Chelkeba L, Melaku T, Tegene E (2018) Pathological Sub Types and Diagnostic Protocols of Stroke among Adult Patients Admitted to Jimma University Medical Center, South West Ethiopia. J Neurol Neurophysiol 9: 466. doi:10.4172/2155-9562.1000466

\section{Conclusion}

The increasing burden of chronic disease in low and middle income countries like Ethiopia poses a challenge to the health care system and the community as whole. In this study the CT scan performance rate was almost for half patients, showing high rate of stroke diagnosis using clinically alone. Using WHO criteria almost equal prevalence of ischemic and hemorrhagic stroke patients showing that the prevalence of hemorrhagic stroke was higher as compared to western countries.

Etiologic investigation for stroke was infrequently performed by the lack of systematic cardiological examinations and brain imaging. Most common site of lesion on hemorrhage stroke was basal ganglia and of ischemic stroke was parietal and cortical lobe. Up on CT scan majority of hemorrhagic stroke and ischemic stroke patients had Intraparenchymal Bleed (IPB) only and right hemisphere involvement, respectively. The laboratory done for the patients in this study was relatively poor because of most patients do not afford and malfunctioning of different equipment's.

Clinical diagnosis of stroke does not always correlate with CT diagnosis. It is therefore, pertinent that CT scan/MRI should be incorporated in stroke management where possible. It was recognized that in our hospital, the diagnostic protocol for stroke patients is suboptimal and lagging behind the recommended guidelines due to lack of appropriate diagnostic agent for etiologic investigation in addition to trained man power in stroke speciality, thus necessary etiologic investigations and trained man power should be available as much as possible to fit the number of stroke patients visiting the hospital. An emergency care set up, perhaps protocol based and capable of early brain imaging is essential through smoothly functioning cooperation of all professionals involved for the early identification of stroke for proper management.

\section{Abbreviations and Acronyms}

CT: Computed Tomography; CVDs: Cardiovascular diseases; ECG: Electrocardiography; GBD: Global Burden of Diseases; HS: Hemorrhagic Stroke; HTN: Hypertension; ICH: Intracerebral Hemorrhage; IHD: Ischemic Heart Disease; IS: Ischemic Stroke; JUMC: Jimma University Medical Center; LMICs: Low and Middle Income Countries; MRI: Magnetic Resonance Imaging; NCDs: NonCommunicable Diseases; RHD: Rheumatic Heart Disease; SSA: Sub Saharan Africa; SU: Stroke Unit; TOAST: Trial of Org 10172 in Acute Stroke Treatment; WHO: World Health Organization.

\section{Acknowledgment and Funding}

We thanks Jimma University for funding and supporting the study. We are grateful to staff members of stroke unit of JUMC, data collectors and study participants for their cooperation in the success of this study. The funding body did not have any role in study design, data collection, analysis, interpretation of data or in writing the manuscript.

\section{Author's Contributions}

GF contributes in the design the study, analysis and write up the manuscript. AK made the data analysis and interpretation of the data. LC and TM contributed to the design of the study, manuscript editing and manuscript review. ET participated in laboratory and imaging data interpretation. All authors read and approved the final version of the manuscript and agree to be accountable for all aspects of the work in ensuring that questions related to the accuracy or integrity of any part of the work are appropriately investigated and resolved.

\section{Ethics Approval and Consent to Participate}

Ethical clearance was obtained from the Institutional Review Board (IRB) of Jimma University, Institute of health. Verbal consent was obtained from responsible bodies of the hospital and stroke unit of the hospital prior to the interview and reviews of the patient data. At hospital patients were informed about the study and verbal consent was obtained. All patients got the right to opt out of the research. The data from the case records and interview was handled with strong confidentiality. Neither the case records nor the data extracted was used for any other purpose. The confidentiality and privacy of patients was assured throughout by removing identifiers from data collection tools using different codes.

\section{Consent for Publication}

Not applicable. No individual person's personal details, images or videos are being used in this study.

\section{References}

1. Moran A, Forouzanfar M, Sampson U, Chugh S, Feigin V, et al. (2013) The epidemiology of cardiovascular diseases in sub-Saharan Africa: The global burden of diseases, injuries and risk factors 2010 study. Progress in cardiovascular diseases 56(3): 234-239.

2. Alemayehu CM, Birhanesilasie SK (2013) Assessment of stoke patients: Occurrence of unusually high number of haemorrhagic stroke cases in tikur anbessa specialized hospital, addis ababa, Ethiopia. Clinical Medicine Research 2(5): 94-100.

3. Patne SV, Chintale KN (2016) Study of clinical profile of stroke patients in rural tertiary health care centre. Int J Adv Med 3(3): 666-670.

4. Dash D, Bhashin A, Pandit AK, Tripathi M, Bhatia R, et al. (2014) Risk factors and etiologies of ischemic strokes in young patients: A tertiary hospital study in north India. Journal of stroke 16(3): 173-177.

5. Tshikwela ML, Londa FB, Tongo SY (2015) Stroke subtypes and factors associated with ischemic stroke in Kinshasa, Central Africa. African health sciences 15(1): 68-73.

6. Kolapo KO, Vento S (2011) Stroke: A realistic approach to a growing problem in sub-Saharan Africa is urgently needed. Tropical medicine \& international health 16(6): 707-710.

7. Adeloye $D$ (2014) An estimate of the incidence and prevalence of stroke in Africa: A systematic review and meta-analysis. PLoS ONE 9(6): e100724.

8. Owolabi MO, Agunloye AM (2013) Which risk factors are more associated with ischemic rather than hemorrhagic stroke in black Africans? Clinical neurology and neurosurgery 115(10): 2069-2074.

9. Owolabi MO, Akarolo-Anthony S, Akinyemi R, Arnett D, Gebregziabher M, et al (2015) The burden of stroke in Africa: A glance at the present and a glimpse into the future. Cardiovascular journal of Africa $26(2)$ : S27-38.

10. Chidiogo O, Chukwuemeka N, Onwuchekwa R, Chinwe R-O, Deborah O, et al. (2015) Computerized tomography and clinical correlation of stroke diagnosis in University of Port Harcourt Teaching Hospital. Journal of Medicine and Medical Sciences 6(5): 90-94.

11. Berkowitz AL, Westover MB, Bianchi MT, Chou SH (2014) Aspirin for acute stroke of unknown etiology in resource-limited settings: A decision analysis. Neurology 83(9): 787-793.

12. Kuriakose C, Shifafiya MN, Tharakan NS, KS, Kumar RS (2016) A prospective study of clinical profile of stroke in a tertiary care hospital. Asian J Pharm Clin Res 9(3): 1-4.

13. Gebremariam SA, Yang HS (2016) Types, risk profiles, and outcomes of stroke patients in a tertiary teaching hospital in northern Ethiopia. eNeurologicalSci 3: 41-47.

14. Deresse B, Shaweno D (2015) Epidemiology and in-hospital outcome of stroke in South Ethiopia. J Neurol Sci 355(1-2): 138-142.

15. Krishnamurthi RV, Feigin VL, Forouzanfar MH, Mensah GA, Connor M, et al. (2013) Global and regional burden of first-ever ischaemic and haemorrhagic stroke during 1990-2010: findings from the global burden of disease study 2010. The Lancet Global health 1(5): e259-281.

16. Bekele A, Kebede $O$ (2002) Stroke admission to tikur anbessa teaching hospital: With emphasis on stroke in the young. Ethiopian Journal of Health Development 16(3): 309-315.

17. Greffie ES, Mitiku T, Getahun S (2015) Risk factors, clinical pattern and outcome of stroke in a referral hospital, Northwest Ethiopia. Clinical Medicine Research 4(6): 182-188.

18. Ekeh B, Ogunniyi A, Isamade E, Ekrikpo U (2015) Stroke mortality and its predictors in a Nigerian teaching hospital. African health sciences 15(1): 74-81. 
Citation: Fekadu G, Chelkeba L, Melaku T, Tegene E (2018) Pathological Sub Types and Diagnostic Protocols of Stroke among Adult Patients Admitted to Jimma University Medical Center, South West Ethiopia. J Neurol Neurophysiol 9: 466. doi:10.4172/2155-9562.1000466

Page 9 of 18

19. Wu CY, Wu HM, Lee JD, Weng HH (2010) Stroke risk factors and subtypes in different age groups: A hospital-based study. Neurology India. 58(6): 863-868.

20. Deleu D, Inshasi J, Akhtar N, Ali J, Vurgese T, et al. (2011) Risk factors, management and outcome of subtypes of ischemic stroke: A stroke registry from the Arabian Gulf. J Neurol Sci 300(1-2): 142-147.

21. World health organization (2006) The WHO step wise approach to stroke surveillance.

22. Tirschwell DL, Ton TG, Ly KA, Van Ngo Q, Vo TT, et al. (2012) A prospective cohort study of stroke characteristics, care, and mortality in a hospital stroke registry in Vietnam. BMC neurology 12: 150

23. de Carvalho JJ, Alves MB, Viana GA, Machado CB, dos Santos BF, et al. (2011) Stroke epidemiology, patterns of management, and outcomes in Fortaleza, Brazil: A hospital-based multicenter prospective study. Stroke 42(12): 3341 3346.

24. Njoku CH, Aduloju AB (2004) Stroke in Sokoto, Nigeria: A five year retrospective study. Annals of African Medicine 3(2): 73-76.

25. Vaidya CV, Majmudar DK (2014) A retrospective study of clinical profile of stroke patients from GMERS Medical College and Hospital, Gandhinagar, Gujarat. Int J Clin Trials 1(2): 62-66.

26. Nkoke C, Lekoubou A, Balti E, Kengne AP (2015) Stroke mortality and its determinants in a resource-limited setting: A prospective cohort study in Yaounde, Cameroon. J Neurol Sci 358(1-2): 113-117.

27. Masood CT, Hussain M, Anis ur R, Abbasi S (2013) Clinical presentation, risk factors and outcome of stroke at a district level teaching hospital. J Ayub Med Coll Abbottabad 25(1-2): 49-51.
8. Desalu OO, Wahab KW, Fawale B, Olarenwaju TO, Busari OA, et al. (2011) A review of stroke admissions at a tertiary hospital in rural Southwestern Nigeria. Annals of African medicine 10(2): 80-85

29. Atadzhanov M, Mukomena P, ShabirLakhi, Ross OA, Meschia JF (2012) Stroke characteristics and outcomes of adult patients. Admitted to the University Teaching Hospital, Lusaka, Zambia. The open general and internal medicine journal 5: 3-8.

30. Sarkar D, Halder S, Saha BK, Biswas P (2016) A study of stroke patients with respect to their clinical and demographic profile and outcome. Int J Res Med Sci 4(9): 4061-4066.

31. Bennour AM, Ehmoda F, Bo-Shaala S. (2014) Clinical characteristics, risk factors, management and outcome of patients admitted with acute stroke in benghazi, libya. Ibnosina J Med BS 6(4): 159-167.

32. Sagui E, M'Baye PS, Dubecq C, Ba-Fall K, Niang A, et al. (2005) Ischemic and hemorrhagic strokes in Dakar, Senegal: A hospital-based study. Stroke 36(9): 1844-1847.

33. Ogeng'o JA, Olabu BO, Mburu AN, Sinkeet SR, Ogeng'o NM (2015) Ischemic Cortical Stroke in a Kenyan Referral Hospital. J Mol Biomark Diagn 5: 238.

34. Jowi JO, Mativo PM (2008) Pathological sub-types, risk factors and outcome of stroke at the Nairobi Hospital, Kenya. East African medical journal 85(12) 572-581.

35. Tan KS, Navarro JC, Wong KS, Huang YN, Chiu HC, et al. (2014) Clinical profile, risk factors and aetiology of young ischaemic stroke patients in Asia: A prospective, multicentre, observational, hospital-based study in eight cities. Neurology Asia 19(2): 117-127. 